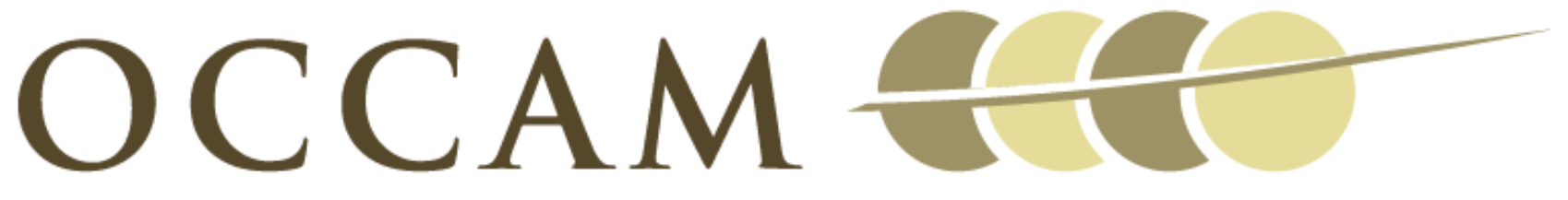

OXFORD CENTRE FOR COLLABORATIVE APPLIED MATHEMATICS

Report Number 12/01

Mechanical growth and morphogenesis of seashells by

Derek E. Moulton, Alain Goriely and Régis Chirat

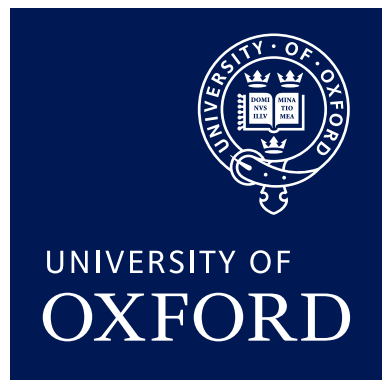

Oxford Centre for Collaborative Applied Mathematics Mathematical Institute 24 - 29 St Giles'

Oxford

OX1 3LB

England 



\title{
Mechanical growth and morphogenesis of seashells
}

\author{
Derek E. Moulton *, Alain Goriely* and Régis Chirat ${ }^{\dagger}$ \\ * OCCAM, Mathematical Institute, University of Oxford, Oxford, UK, and † CNRS 5276, Université Lyon 1, 69622 Villeurbanne Cedex, France \\ Submitted to Proceedings of the National Academy of Sciences of the United States of America
}

\begin{abstract}
Seashells grow through the local deposition of mass along the aperture. Many mathematical descriptions of the shapes of shells have been provided over the years, and the basic logarithmic coiling seen in mollusks can be simulated with few parameters. However, the developmental mechanisms underlying shell coiling are largely not understood and the ubiquitous presence of ornamentation such as ribs, tubercles, or spines presents yet another level of difficulty. Here we develop a general model for shell growth based entirely on the local geometry and mechanics of the aperture and mantle. This local description enables us to efficiently describe both arbitrary growth velocities and the evolution of the shell aperture itself. We demonstrate how most shells can be simulated within this framework. We then turn to the mechanics underlying the shell morphogenesis, and develop models for the evolution of the aperture. We demonstrate that the elastic response of the mantle during shell deposition provides a natural mechanism for the formation of three-dimensional ornamentation in shells.
\end{abstract}

growth | mechanics | pattern formation | seashell

Abbreviations: CDRT, Coiling Dilation, Rotation, Translation

S eashells have intrigued scientists for centuries, in particular mathematicians have long appreciated their beautiful patterns, regularity, and self similarity. Yet underlying the elegant mathematical structure of the shape of shells is a growth and development process which is still poorly understood. For instance, while logarithmic coiling in shells was first mathematically described over a century ago [1], the developmental bases of these shell forms remains unknown. Similarly, the three-dimensional ornamentations on shells, which can be seen as a deviation from simple coiling, emerge through largely unknown processes. Such issues fall into the general problem of connecting the form of biological structures to the underlying forces and mechanisms behind growth and development processes [2]. Seashells, and mollusk shells in particular, provide an advantageous case study for this problem due to their aesthetic appeal, their large variety and fossil record, and the relative simplicity of the growth process, whose history is recorded in the shell shape.

There are various objectives in studies and models of shells. Much of the early work was concerned with characterising the diversity of shells in terms of morphological parameters $[3,4,5,6,7,8]$. While these models were able to describe the shape of shells, and sometimes provide very realistic representations, they were disconnected from the growth process. A number of studies have since tried to connect shell shape to relative and absolute growth rate [9], notably through growth vector models $[10,11]$; however these representations often have the drawback of needing a large parameter space and/or rely on geometric constructions disconnected from the underlying growth process. In terms of the mechanisms behind the patterns and form of shells, previous modeling approaches have focussed on activator-inhibitor systems [8, 12], and neural based explanations [13], and although the genesis of biological form is proximately a matter of forces, few studies have addressed the mechanical basis of shell morphogenesis $[14,15,16,17,18]$.

In this paper, we first present a framework to study and simulate shell growth, by generalising a model for surface growth [19]. The descriptions is focussed on the local environment of the shell aperture, where growth occurs. Shell growth can be simulated for arbitrary and evolving aperture shape and with a minimal number of parameters. The basic idea is that the evolution of a shell can be seen as the result of two complementary processes. In one process, the shell aperture undergoes coiling, dilation, translation, and rotation (CDRT). This process forms the basic macroscopic shell form that is typically very regular. That is, these rates are highly controlled throughout the shell development, and the resulting shell form has the mathematical regularity (self-similarity, e.g.) that has attracted so much attention. In the second process, the aperture shape evolves, producing ornamentation such as ribs or spines on the shell surface. The evolution of the aperture is governed by forces underlying shell morphogenesis, and an understanding of the growth process is therefore necessary. Hence, as a second step we develop a model for the evolution of the aperture based on the mechanical environment of the shell deposition process. In mollusks, the shell is secreted at the level of the aperture by a thin soft tissue, the mantle, modelled here as an elastic tissue. In our model, the mechanical response of the mantle governs the evolution of the aperture, which is then coupled to the CDRT kinematics.

The organisation of this paper is as follows. First, we outline the mathematical and computational basis of our approach to shell growth. Within this framework, essentially any form of shell can be generated. We then incorporate mechanics in the growth process and provide a biomechanical basis for ornamentation in shells. We produce two models for commarginal and antimarginal ornamentation that govern the evolution of the aperture in the general growth framework.

\section{Shell growth via curve evolution}

Shell growth is modelled via the evolution of a generating curve representative of the shell aperture [19]. At each point on the generating curve a local vector field is defined that dictates the direction and rate of accretion. Through this process, a surface $\mathbf{r}(\sigma, t)$ is created. Here $\sigma$ is a material parameter, so that at any time $t_{0}$, the space curve $\mathbf{r}\left(\sigma, t_{0}\right)$ is the aperture at that time (see Figure 1a) . The main difference between this and other growth models, [20,11], is that growth is described in a local basis attached to each point of the aperture, and not on a fictitious centerline [21, 22, 23, 24]. Here, by attaching a frame at each point of the aperture, growth is described entirely in terms of the local geometry of the aperture, which apart from its mathematical elegance [19], reduces the

\section{Reserved for Publication Footnotes}


construction of shell shapes to a small number of parameters that can be directly related to the growth process.

For the majority of seashells, the aperture remains planar or very nearly planar, and for simplicity we restrict our attention to arbitrary planar curves. A convenient choice of frame is the standard Frenet frame, where the local basis vectors are the familiar (normal,binormal,tangent) $=(\boldsymbol{\nu}, \boldsymbol{\beta}, \boldsymbol{\tau})$. Growth is thus dictated by three quantities, $\mathrm{v}_{\nu}, \mathrm{v}_{\beta}, \mathrm{v}_{\tau}$, which represent the growth velocity in the normal, binormal, and tangent directions, respectively. Given these functions, the evolution of the curve is dictated by

$$
\dot{\mathbf{r}}(\sigma, t) \equiv \partial_{t} \mathbf{r}=\mathbf{v}=\mathbf{v}_{\nu}(\sigma, t) \boldsymbol{\nu}+\mathbf{v}_{\beta}(\sigma, t) \boldsymbol{\beta}+\mathbf{v}_{\tau}(\sigma, t) \boldsymbol{\tau} .
$$

Equation [1] is coupled to a description of how the frames vary in time and space. A full derivation of the system is given in [19] and summarized in Appendix A. The general solution in local coordinates gives rise to 4 basic transformations of the aperture under which the aperture shape is fixed. These are coiling, dilation, rotation, and translation, which we abbreviate CDRT. Many surfaces can be generated within this context, and realistic seashells can be created with simple forms of the local velocity components $\mathrm{v}_{\nu}, \mathrm{v}_{\beta}, \mathrm{v}_{\tau}$. Here, we extend this model to arbitrary local velocity rules, and more importantly we allow the shape of the aperture to change. For an arbitrary curve evolution, the governing system is not analytically tractable, a discrete time-step formalism is needed. This requires proper descriptions of all geometric quantities in a discrete environment, using the tools of discrete differential geometry [25].

Key to obtaining a framework for a general evolution, and a fundamental idea in this work, is the notion that shell growth can be described via two separate processes: CDRT, which does not change the aperture shape, and the evolution of the aperture, which is driven by underlying biomechanical forces (see Figure 1b). We capture this idea in the curve evolution framework in three steps.

The first step is to determine the rules for CDRT in a discrete setting, corresponding to the analytical rules found in the continuous case [19]. (Details are given in Appendix B.) Within this class of a fixed aperture shape, a wide variety of shells can be generated, as shown in Figure 2.

The second step is to allow the shape of the aperture to evolve (in between CDRT steps). That is, we consider arbitrary velocities in the tangent and normal directions (assuming the curve remains planar, an arbitrary velocity in the binormal direction is not permissible). This has to be done carefully, as all local quantities on the curve must be appropriately updated. For details, see Appendix C.

To complete the formulation, we combine CDRT with shape evolution. The idea is that shape change can be seen as an added feature imposed on a shell with an aperture evolving with fixed CDRT. Computationally, the key to this process is to evolve two curves, the actual aperture embedded in $\mathcal{R}^{3}$ (i.e. the curve $\mathbf{r}$ that satisfies [1]), which traces out the full surface, and a fixed plane reference curve $\hat{\mathbf{r}}$ that tracks only the evolution of the aperture shape. This process is easily implemented within a local formulation, as the local basis on each curve provides the information necessary to map from the fixed plane curve $\hat{\mathbf{r}}$ to the actual curve $\mathbf{r}$. See Appendix D for details.

A key benefit of the local formulation is that complex surfaces may be simulated with a small number of parameters, as demonstrated in Figure 3. These surface are generated as follows: first, an aperture shape and 4 CDRT parameters are chosen for the basic evolution without ornamentation. The ornamentation is then added as a second feature. In Figure $3 \mathrm{~b})$, for example, the spines are generated with a 5 param- eter shape change velocity. Explicit formulas are given in Appendix F.

\section{Mechanics and ornamentation}

The formulation we have described thus far is capable of generating a great variety of forms found in nature. However, so far this is only a geometric description. Central to this paper is a physical basis for the evolution of the aperture based on the mechanics underlying the deposition process. While several papers with reaction diffusion or neural based models have reproduced pigmentation patterns in shells [13, 8, 12], a thorough mechanism for ornamentation has not been given. The role of mechanics in the genesis of three-dimensional ornamentation has been proposed on the basis of empirical observations $[14,15,18]$ as well as in theoretical papers $[16,17]$, but never integrated into a three-dimensional mechanical perspective. Mechanics was only implicitly included in [16], and while the work of Morita [17] is perhaps the only previous theoretical work that fully addresses shell growth from a mechanical perspective, the elastic DMS tube model does not really take into account accretionary growth.

The basic idea of our approach is as follows: molluskan shells are formed by the deposition of material by a soft, skirtlike tissue called the mantle. At the time of shell growth, the mantle adheres at the shell aperture and adds a layer of shell material. The aperture acts as a sort of rigid template for the mantle, potentially inducing stresses and strains that ultimately affect the shape of the new layer and thus the evolution of the shell. Modeling the mantle within the theory of elastic rods, we demonstrate below how this universal process can lead to the formation of a variety of three-dimensional ornamentation.

Commarginal ribs. Commarginal, or transverse ribs, are a common feature in a number of mollusk shells, characterized by an oscillatory component to the dilation of the aperture. We model the mantle as a circular growing elastic ring with a given growth rate. When the mantle adheres to the current shell edge, it is subject to a compression/stretching force, and an attachment force. We assume that the mantle remains circular, and that the equilibrium diameter is a balance between these two forces. In equilibrium, the mantle is not in a stressfree state, and we assume that the deposition of new layer is controlled by the level of stress through an adjustment of the angle of deposition (see Figure 4). Specifically, if the mantle is in compression it increases the angle of deposition so to increase the radius of the new aperture and therefore relieve its stress; put simply, if the mantle "feels crowded", it tries to build a bigger shell. Conversely, if the mantle is in tension, it does the opposite, decreasing the angle, thereby decreasing the rate of increase of aperture radius.

The setup for the model is pictured in Figure 4 . Let $k_{g}$ be the growth rate, i.e. in time $\Delta t, k_{g} \Delta t$ is the length of material added to the shell. We will assume $k_{g}$ to be a constant. ${ }^{1}$ The angle of material deposition is denoted by $\phi$. Denoting $r_{s}$ as the shell radius, it follows that

$$
\dot{r}_{s}=\frac{d r_{s}}{d t}=k_{g} \cos \theta \sin \phi
$$

\footnotetext{
${ }^{1}$ When coiling is included, $k_{g}$ will vary along the aperture, since the length of material added in the binormal direction is non-uniform (see Appendix B). In this case, we define $k_{g}$ to be the point on the aperture where the growth rate is maximum, and still take it to be constant in time. The growth rate at every other point is subsequently determined based on the coiling parameters.
} 
where $\theta$ is the fixed angle of the growth vector between the normal and tangent direction, and thus describes the amount of rotation. The key assumption is that the mantle can change the angle $\phi$ based on its stress level. Let $n_{3}$ be the axial stress in the mantle edge; note $n_{3}>0$ if the mantle is in tension and $n_{3}<0$ if the mantle is in compression. This axial stress $n_{3}$ depends on the shell radius $r_{s}$ as well as the diameter of the mantle edge in the unstressed state, determined by the growth function $\gamma$. The value of $n_{3}$ is found by computing the equilibrium diameter of the mantle edge for given shell radius. Details are provided in Appendix E. The general system is

$$
\begin{aligned}
\frac{d r_{s}}{d t} & =k_{g} \cos \theta \sin \phi \\
\frac{d \phi}{d t} & =\mathcal{H}\left(n_{3}\left(r_{s}, \gamma\right)\right),
\end{aligned}
$$

where the function $\mathcal{H}\left(n_{3}\left(r_{s}, \gamma\right)\right)$ describes the feedback of the mantle, and the growth function $\gamma=\gamma(t)$ is taken as input. For given functions $\mathcal{H}$ and $\gamma$ and initial values $r_{s}(0)$ and $\phi(0)$, the system of ordinary differential equations [3] can easily be solved numerically. We consider 3 forms for $\mathcal{H}$, and plot the resulting shell radius $r_{s}(t)$ in Figure 5. In Figure 5a, a simple linear law is used,

$$
\mathcal{H}_{1}=-k_{\phi} n_{3} .
$$

In this case, a fairly uniform oscillation of the shell radius is produced. In many species of rib producing shell, in particular ammonites, the oscillation is not uniform but rather consists of wide valleys and sharp ridges. This suggests that the mantle responds more strongly to tension than to compression. A feedback law that takes this into account is

$$
\mathcal{H}_{2}=k_{\phi}\left(1-e^{K n_{3}}\right) .
$$

With this form, the change in growth angle $\phi$ is greater when the mantle is in tension. The radius $r_{s}(t)$ for this form is shown in Figure 5b. Here we see the expected effect of sharp ridges and wide valleys. However, this still does not quite capture the pattern seen in most rib producing shells, because the wavelength is nearly constant throughout development, whereas for instance in ammonites the wavelength of the ridges increases with time. This may be due to the increasing thickness of the mantle throughout development, that is a thicker membrane may be less easily incrementally "reoriented" than a thin one. This leads us to consider a third form,

$$
\mathcal{H}_{3}=\frac{k_{\phi}}{r_{s}}\left(1-e^{K n_{3}}\right) .
$$

Here, the feedback is still stronger when the mantle is in tension, but the feedback decreases through development as the mantle grows. The radius for this form is plotted in Figure 5c. In this case, the wavelength of the ridges increases with time; interestingly, the amplitude does as well which is consistent with observations in ammonites.

The system [3] governs the evolution of the aperture - in this case, the amount of dilation - based on the mechanical environment of the mantle. Seashell growth can be simulated by combining this with CDRT. To simulate an ammonite, a fixed rule for coiling is given, the oscillatory dilation is governed by [3] and the feedback law by [6]. The result, plotted in Figure 6, shows a realistic ammonite displaying a typical ribbing pattern.

Antimarginal ornamentation. Antimarginal ornamentation are patterns orthogonal to the aperture. This includes antimarginal ribs as well as more complex patterning such as spines and fractal-like structures. The basic mechanism we have proposed for commarginal ornamentation, i.e. that the elastic response of the mantle adhering to the aperture dictates the evolution, can explain antimarginal ornamentation as well. Here, we extend a hypothesis previously proposed based on empirical observations in bivalve shells [26]. The idea is as follows: since the growing mantle will exhibit an excess of length compared to the previous growth increment, it will be subject to a buckling force upon adhering to the aperture. Thus, whereas in the previous subsection we assumed the mantle had a circular shape and evolved via stress feedback, here we allow the shape of the mantle to deform as well. The deposited layer of shell material takes the deformed shape, and thus forms the template for the next growth increment. In this manner, the mechanical deformation of the mantle dictates the evolution of the shape of the aperture.

The model for the deformation of the mantle is shown in Figure 7. As before, the mantle edge is treated as a planar elastic rod that attaches to a rigid foundation (the current aperture) through an elastic spring force. For simplicity and to isolate the effect of the deforming mantle and hence the evolving aperture, we assume an inextensible rod. The centerline of the mantle is the curve $\hat{\mathbf{r}}(\sigma)$ with arclength $L$ and the centerline of the aperture is $\mathbf{f}(\sigma)$ with arclength $L_{0}$. It is assumed that $L>L_{0}$ to account for growth of the mantle. The shape of the mantle is determined as the curve that minimizes the sum of the bending energy and the foundation energy, subject to the given length constraint. The foundation energy takes the form

$$
\mathcal{E}_{f}=\frac{k_{s}}{2} \int_{0}^{L}|\hat{\mathbf{r}}-\mathbf{f}|^{2} d \sigma
$$

were $k_{s}$ is a stiffness parameter for the foundation. The bending energy depends on the curvature $\kappa$ of the mantle,

$$
\mathcal{E}_{b}=\frac{\mathrm{EI}}{2} \int_{0}^{L} \kappa^{2} d \sigma,
$$

where EI is the bending stiffness (the product of Young's modulus and the second moment of area).

As described in the appendix, shape evolution is coupled to CDRT by evolving two curves, the actual curve embedded in $\mathcal{R}^{3}$ and a fixed plane curve $\hat{\mathbf{r}}$ that tracks only the evolution of the aperture. Suppose at time $t$ this curve is given by $\hat{\mathbf{r}}_{t}$, and that after the mantle deformation the curve is given by $\hat{\mathbf{r}}_{t+\Delta t}$, determined as the minimizer of the total energy $\mathcal{E}=\mathcal{E}_{f}+\mathcal{E}_{b}$. The deformation defines a velocity $\mathbf{q}=\hat{\mathbf{r}}_{t+\Delta t}-\hat{\mathbf{r}}_{t}$, from which the local velocities $\mathrm{q}_{\nu}$ and $\mathrm{q}_{\tau}$ can be computed. It should be noted that while coiling, rotation, and translation have no effect on the mantle buckling, dilation plays a role as it increases the total length of the aperture; hence the dilation step must be incorporated within the shape change evolution. Each growth increment, representing one physical growth step, consists of the following 4 steps, illustrated in Figure 8:

Ia. The full curve undergoes CDRT.

Ib. The fixed plane curve undergoes dilation only.

IIa. Mantle growth leads to an increase in the arclength of the aperture. The deformed mantle is computed via energy minimization. The fixed plane curve is evolved.

IIb. The full curve is correspondingly evolved through translation of the velocity into local coordinates.

\section{Example - bivalve}

As an example, we simulate the growth of a bivalve shell. The initial foundation is taken to be a semi circle, with a fixed increase in length occurring at each stage of mantle growth. 
Fixed rates of coiling, dilation, and translation are imposed with the shape change. Figure 9 shows the full surface produced via the evolution, as well as the evolution of half of the fixed plane curve; it can be noted that the folds of the aperture deepen over time through the incremental mantle deformation process.

\section{Discussion}

We have implemented a general model for the growth and morphogenesis of seashells. The underlying mathematical framework derived in Ref. [19] was extended to a discrete setting where it could be applied to an arbitrary evolution. Key to the formulation was the decomposition of growth to a non-shape-changing step consisting of CDRT; and a step in which the aperture shape evolves. Computationally, the implementation of this decomposition relies on a description in terms of local variables. The evolution of the aperture shape may in general be governed by mechanical, biological, chemical, or environmental forces. The framework developed is well suited to investigate any of these inputs. This approach is also applicable for other surface growth processes. In this paper, we have focussed specifically on mechanical forces, on the premise that biological morphogenesis is a mechanical process that connects genetic, molecular, and cellular activities with the macroscopic deformations shaping organisms.

In particular, we considered two related models coupling the mechanical environment of the mantle to the growth kinematics, and explored the formation of three-dimensional ornamentation in shells. The soft mantle edge, where shell deposition occurs, was modeled as an elastic rod, and the forces imposed on the mantle due to adhering to the aperture governed the shape of the mantle and hence the evolution of the aperture. In the first model, commarginal ribs were produced by assuming a circular mantle with control over the amount of dilation based upon the stress induced by attachment to the aperture. In the second model, the buckling of the mantle edge dictated the incremental change in shape of the aperture, thereby simulating the formation of antimarginal ribs. In each case, we demonstrated how the mechanics driven shape evolution could be coupled to CDRT to explain realistic shells from simple physical principles.

The characteristic of mollusk shells is that the shape is generated at the level of a moving boundary, the form taken by the secreting mantle along the growing shell front being fixed in the calcified edge, while this patterned calcified edge biases the configuration of the secreting mantle at the next growth increment. This dynamic shows that far from being only a passive recorder of processes taking place within the secreting membrane, the shell is in fact involved in the morphogenesis of its own ornamentation. Theoretical studies based on reaction-diffusion systems do not integrate this dynamic, because within these models the shell is no more than a record of a chemical pattern in the secreting mantle edge. Within our framework we can investigate the correlation between the general shell geometry and a range of ornamentation. Particularly, because real mollusk shells are threedimensional curved objects, one possible influencing factor is the curvature of the growing front. Mechanics also plays an important role in the emergence of spines that correspond to a more elaborate form of antimarginal ornamentation generated in Figure 9. The morphogenesis of these structures is explored in depth in a separate paper [27], where it is shown that they can emerge as a consequence of the same basic growth-induced buckling instability. Interestingly, these structures are prevalent in a number of mollusk species and may be associated ei- ther with commarginal or antimarginal ornamentation. Given the similarity in mechanism between these two distinct forms of ornamentation, it is natural to ask whether in fact each of these forms can be produced with different parameter regimes within a single mechanical model. Within such a theory, shell morphogenesis can be thought of as a trajectory unfolding in a shape space constrained by geometry and mechanics. This approach provides a new, far-reaching perspective of the phenotypic evolution of the shells of the second largest phylum in the animal kingdom. 


\section{Appendix: A. Continuous system for surface growth kine- matics}

In this appendix we provide the governing equations for the curve evolution in the continuous case. Consider an initial curve $\mathbf{r}(\sigma, 0)$, where $\sigma \in[0, L]$ is the material parameter (not necessarily arclength), equipped with the orthonormal Frenet basis $\mathbf{D}=(\boldsymbol{\nu}, \boldsymbol{\beta}, \boldsymbol{\tau})$. The tangent vector $\boldsymbol{\tau}$ is related to the spatial derivative of $\mathbf{r}$ by a stretch factor $\lambda$,

$$
\mathbf{r}^{\prime} \equiv \partial_{\sigma} \mathbf{r}=\lambda \boldsymbol{\tau}
$$

Recall the Frenet-Serret formulas

$$
\begin{aligned}
& \boldsymbol{\nu}^{\prime}=\mathrm{u}_{3} \boldsymbol{\beta}-\mathrm{u}_{2} \boldsymbol{\tau} \\
& \boldsymbol{\beta}^{\prime}=-\mathrm{u}_{3} \boldsymbol{\nu} \\
& \boldsymbol{\tau}^{\prime}=\mathrm{u}_{2} \boldsymbol{\nu} .
\end{aligned}
$$

Here $\mathrm{u}_{2} / \lambda$ and $\mathrm{u}_{3} / \lambda$ are the geometric curvature and torsion, respectively. For a general basis, there is also a third component $\mathbf{u}_{1}$, which completes the axial vector $\mathbf{u}=\left(\mathbf{u}_{1}, \mathbf{u}_{2}, \mathbf{u}_{3}\right)$, but in the Frenet basis $\mathbf{u}_{1}=0$. Time derivatives of the frame can be expressed in terms of the frame itself via the axial vector $\mathrm{w}=\left(\mathrm{w}_{1}, \mathrm{w}_{2}, \mathrm{w}_{3}\right)$ as

$$
\begin{aligned}
& \dot{\boldsymbol{\nu}}=\mathrm{w}_{3} \boldsymbol{\beta}-\mathrm{w}_{2} \boldsymbol{\tau} \\
& \dot{\boldsymbol{\beta}}=-\mathrm{w}_{3} \boldsymbol{\nu}+\mathrm{w}_{1} \boldsymbol{\tau} \\
& \dot{\boldsymbol{\tau}}=\mathrm{w}_{2} \boldsymbol{\nu}-\mathrm{w}_{1} \boldsymbol{\beta} .
\end{aligned}
$$

The curve evolution is governed by a growth velocity vector field $\mathbf{v}$, defined in terms of the local basis via

$$
\dot{\mathbf{r}}=\mathbf{v}=\mathrm{v}_{\nu} \boldsymbol{\nu}+\mathrm{v}_{\beta} \boldsymbol{\beta}+\mathrm{v}_{\tau} \boldsymbol{\tau},
$$

where the velocity components $\left(\mathbf{v}_{\nu}, \mathbf{v}_{\beta}, \mathbf{v}_{\tau}\right)$ are continuous functions of $\sigma$ and $t$. Assuming that $\mathbf{r}(\sigma, t)$ is at least twice differentiable in $\sigma$ and $t$, we have two sets of compatibility conditions,

$$
\begin{aligned}
& \partial_{\sigma}\left(\partial_{t} \mathbf{r}\right)=\partial_{t}\left(\partial_{\sigma} \mathbf{r}\right) \\
& \partial_{\sigma}\left(\partial_{t} \mathbf{D}\right)=\partial_{t}\left(\partial_{\sigma} \mathbf{D}\right),
\end{aligned}
$$

which in component form simplifies to the following system

$$
\begin{aligned}
& \mathrm{v}_{\nu}^{\prime}+\mathrm{u}_{2} \mathrm{v}_{\tau}-\mathrm{u}_{3} \mathrm{v}_{\beta}=\lambda \mathrm{w}_{2} \\
& \mathrm{v}_{\beta}^{\prime}+\mathrm{u}_{3} \mathrm{v}_{\nu}=-\lambda \mathrm{w}_{1} \\
& \mathrm{v}_{\tau}^{\prime}+\mathrm{u}_{1} \mathrm{v}_{\beta}-\mathrm{u}_{2} \mathrm{v}_{\nu}=\dot{\lambda} \\
& -\mathrm{w}_{1}^{\prime}=\mathrm{u}_{2} \mathrm{w}_{3}-\mathrm{u}_{3} \mathrm{w}_{2} \\
& \dot{\mathrm{u}}_{2}-\mathrm{w}_{2}^{\prime}=\mathrm{u}_{3} \mathrm{w}_{1}-\mathrm{u}_{1} \mathrm{w}_{3} \\
& \dot{\mathrm{u}}_{3}-\mathrm{w}_{3}^{\prime}=-\mathrm{u}_{2} \mathrm{w}_{1} .
\end{aligned}
$$

For a given velocity, these equations form a set of 6 nonlinear first order partial differential equations for the 6 dependent variables $\mathbf{u}, \mathbf{w}, \lambda$. Once $\mathbf{u}(\sigma, t)$ and $\lambda$ are known, the surface built through the accretive process can be obtained by integrating the Frenet equations [10] together with [9]. Alternatively, the surface may be obtained from $\mathbf{w}(\sigma, t)$ and $\lambda$ by integrating [11] together with [12].

\section{Appendix: B. Discrete CDRT}

With a discrete, finite time step, the velocity rules derived in [19] for CDRT must be adapted. In this appendix we describe the procedure for converting the continuous rules for CDRT to the discrete environment. We consider an arbitrary planar curve that evolves such that it remains planar at all times. This implies that $\mathrm{u}_{3}=0$. Let the material parameter $\sigma \in[0, L]$ be discretised with $N$ points so that $\sigma_{i}=i \Delta \sigma$ with $\Delta \sigma=L / N$, and time discretised as $t_{j}=j \Delta t$ where $\Delta t$ is the discrete time step. Then the discrete surface is defined by the points $\mathbf{r}_{i}^{j}=\mathbf{r}\left(\sigma_{i}, t_{j}\right)$. Each point also has the accompanying data:
1. A set of three vectors $\left(\boldsymbol{\nu}_{i}^{j}, \boldsymbol{\beta}_{i}^{j}, \boldsymbol{\tau}_{i}^{j}\right)$, i.e. the local frame ${ }^{2}$.

2. A stretch factor $\lambda_{i}^{j}$, corresponding to the continuous definition $\partial_{\sigma} \mathbf{r}=\lambda \boldsymbol{\tau}$.

3. The quantity $\mathbf{u}_{2}^{j}$, the only non-vanishing component of the axial vector in the Frenet basis, which describes the rotation of the frame along the curve about the binormal. The product $\lambda \mathrm{u}_{2}$ is the geometric curvature.

Without loss of generality (WLOG), let the initial curve be set in the $x-y$ plane,

$$
\mathbf{r}_{0}(\sigma)=\mathbf{r}(\sigma, 0)=\left[x_{0}(\sigma), y_{0}(\sigma), 0\right]
$$

Hence the binormal only has a component in the $z$ direction, and the normal and tangent can be expressed in Cartesian coordinates as

$$
\boldsymbol{\nu}(\sigma)=\left[\nu_{x}, \nu_{y}, 0\right], \quad \boldsymbol{\tau}(\sigma)=\left[\tau_{x}, \tau_{y}, 0\right] .
$$

We derive below rules for the discrete evolution of the curve. The key is that the shape does not change. Thus, we need only determine the velocity rules for the first time step, the same rules remain valid at each time step thereafter even though the curve is at a different location in space. This is a benefit of describing the velocity in terms of local frames.

Coiling. In the continuous case, coiling is obtained with a velocity only in the binormal direction, and a linear function of distance along a growth axis [19]. WLOG, let the growth axis be the $x$-axis. Then coiling requires the velocity

$$
\mathrm{v}_{\beta}=b_{1}+b_{2} x_{0}
$$

where $b_{1}$ and $b_{2}$ are constants. ${ }^{3}$ To find the analogue for the discrete case, we must add correction terms to the velocity to ensure that the shape does not change. This is accomplished as follows. Let $\mathbf{v}_{\nu c}$ and $\mathbf{v}_{\tau c}$ denote the correction terms in the normal and tangential directions. In determining the correction terms, we first must adjust the binormal velocity [23], since the correction terms will affect the distance to the coiling axis. Defining

$$
x_{n e w}=x_{0}+\left(\mathrm{v}_{\nu c} \nu_{x}+\mathrm{v}_{\tau c} \tau_{x}\right) \Delta t,
$$

the binormal velocity becomes $\mathrm{v}_{\beta}=b_{1}+b_{2} x_{\text {new }}$.

After a finite time step $\Delta t$ the points on the $y$-axis must remain on the $y$-axis and at the same $y$-value, i.e. they require no correction (if these points had correction terms, twist would be introduced into the finite coiling step). This is illustrated in Figure 10 - the point $a_{\Delta t}$ must be located directly above the point $a_{0}$ at a distance $b_{1} \Delta t$. In the same way, if we defined the origin as the center of the initial curve, then the center at time $\Delta t$ will be at the point $\left[0,0, b_{1} \Delta t\right]$, denoted $c$ in Figure 10. In this way, we can use these two points as anchors for the discrete step.

For every other point, we require that the distance to the center at time $\Delta t$ is equal to the initial distance, and that the angle between the point, the center, and the point $a$ does not

\footnotetext{
${ }^{2}$ Actually, computationally it is more convenient to define the frame in a slightly different way. Note that if the curvature changes sign, then the binormal switches discontinuously from $\mathbf{e}_{z}$ to $-\mathbf{e}_{z}$ and the normal will discontinuously change direction as well. To avoid such discontinuities, we define the frame such that $\beta$ is the same vector at all points on the curve. Strictly speaking, this is not the Frenet frame, however for convenience we will continue to use the notation $(\boldsymbol{\nu}, \boldsymbol{\beta}, \boldsymbol{\tau})$ to describe the local frame.

${ }^{3}$ It is occasionally desirable to have the scaling factor $\lambda$ present (see [19]). The following discussion is easily modified to account for such a term.
} 
change after the finite step. These two conditions define a set of equations which can be solved for $\mathbf{v}_{\nu c}$ and $\mathbf{v}_{\tau c}$. We obtain, after some algebra,

$$
\begin{aligned}
\mathrm{v}_{1 c} & =\frac{\left(g^{-1 / 2}-1\right) \tau_{y} x_{0}}{\hat{d} \Delta t} \\
\mathbf{v}_{3 c} & =-\frac{\left(g^{-1 / 2}-1\right) \nu_{y} x_{0}}{\hat{d} \Delta t},
\end{aligned}
$$

where $g:=1+b_{2}^{2} \Delta t^{2}$ and $\hat{d}:=\tau_{y} \nu_{x}-\tau_{x} \nu_{y}$. The binormal velocity then simplifies to $\mathrm{v}_{\beta}=b_{1}+b_{2} x_{0} g$.

The correction terms ensure that the shape of the curve does not change during the coiling step. It is also necessary to properly update the frames after the finite step. To do this, the binormal is first updated to the normal direction of the plane of the new curve. The tangent and normal vectors are then rotated to match the rotation of the binormal.

Dilation. In the continuous case and for the curve given by Equation [21] in the $\mathrm{x}-\mathrm{y}$ plane, dilation is achieved with a velocity of the form

$$
\mathbf{v}_{d}=c r_{0} \mathbf{e}_{\mathbf{r}},
$$

where $c$ is independent of $\sigma, r_{0}=\left|\mathbf{r}_{0}\right|$, and $\mathbf{e}_{\mathbf{r}}$ is the radial unit vector in the $x-y$ plane. This is converted to the local basis by decomposing $r_{0} \mathbf{e}_{\mathbf{r}}=\alpha_{1} \boldsymbol{\nu}+\alpha_{3} \boldsymbol{\tau}$, so that the local velocities are

$$
\mathrm{v}_{\nu}=c \alpha_{1}, \quad \mathbf{v}_{\tau}=c \alpha_{3} .
$$

In this case, no correction terms are needed. The curve is updated as $\mathbf{r}_{\Delta t}=\mathbf{r}_{0}+\Delta t\left(\mathbf{v}_{\nu} \boldsymbol{\nu}+\mathbf{v}_{\tau} \boldsymbol{\tau}\right)$, and the scaling factor $\lambda$ is updated as $\lambda_{\Delta t}=(1+c t) \lambda_{0}$. The frames do not change with the dilation step.

Rotation. Rotation has a similar form to dilation, but with velocity in the circumferential direction, namely

$$
\mathbf{v}_{r}=b r_{0} \mathbf{e}_{\theta} .
$$

In the same manner as dilation, we decompose $r_{0} \mathbf{e}_{\theta}=\gamma_{1} \boldsymbol{\nu}+$ $\gamma_{3} \tau$. For a discrete rotation step, however, we must add a correction term in the radial direction. That is, the velocity is $b r_{0} \mathbf{e}_{\theta}-v_{c} r_{0} \mathbf{e}_{\mathbf{r}}$, where $v_{c}$ is to be determined. This is illustrated in Figure 11. Imposing the requirement that the radius of any point does not change during the rotation step, a simple geometrical calculation yields the formula

$$
v_{c}=\left(1-\sqrt{1-b^{2} \Delta t^{2}}\right) / \Delta t,
$$

and thus the local velocities are

$$
\mathrm{v}_{\nu}=b \gamma_{1}-v_{c} \alpha_{1}, \quad \mathrm{v}_{\tau}=b \gamma_{3}-v_{c} \alpha_{3} .
$$

The frames undergo a rotation about the binormal. From Figure 11 , it can be seen that the rotation angle is $\theta=\sin ^{-1}(b \Delta t)$.

Translation. The final form of growth within the context of the aperture shape remaining fixed is a global translation. That is, a velocity $\mathbf{v}_{g}(t)$, a velocity in the same direction at all points on the generating curve. Such a velocity corresponds to a pure translation, and requires no correction steps nor frame updating. In the continuous case, the only step needed is to decompose the velocity into local components, i.e. write

$$
\mathbf{v}_{g}=\mathbf{v}_{\nu} \boldsymbol{\nu}+\mathbf{v}_{\beta} \boldsymbol{\beta}+\mathbf{v}_{\tau} \boldsymbol{\tau} .
$$

In the discrete setting, the decomposition is not even necessary and the curve can be immediately updated as $\mathbf{r}_{t+\Delta t}=$ $\mathbf{r}_{t}+\mathbf{v}_{g} \Delta t$.

\section{Appendix: C. Discrete shape evolution}

Here we describe the procedure for the discrete evolution of the shape of the curve. Along with initial curve $\mathbf{r}_{0}(\sigma)=\left[x_{0}(\sigma), y_{0}(\sigma), 0\right]$ and frames $\boldsymbol{\nu}_{0}(\sigma)=\left[\nu_{x}, \nu_{y}, 0\right]$, $\boldsymbol{\tau}_{0}(\sigma)=\left[\tau_{x}, \tau_{y}, 0\right]$, we also assume that we have the initial data $\lambda_{0}(\sigma)=\left|\partial \mathbf{r}_{0} / \partial \sigma\right|=\left|\mathbf{r}_{0}^{\prime}\right|$ as well as the value $\mathbf{u}_{2_{0}}=\lambda_{0} \kappa_{0}$, where $\kappa$ is the geometric curvature. Note that $\mathrm{u}_{2}$ describes the rotation in space of the local frame about the binormal.

Let the evolution of the curve be dictated by the velocity

$$
\mathbf{q}=\mathbf{q}_{\nu} \boldsymbol{\nu}+\mathbf{q}_{\tau} \tau
$$

where the $\mathbf{q}_{i}$ are arbitrary functions of $\sigma$ and $t$. After time step $\Delta t$, the curve is $\mathbf{r}_{\Delta t}=\mathbf{r}_{0}+\left(\mathbf{q}_{\nu_{0}} \boldsymbol{\nu}_{0}+\mathbf{q}_{\tau_{0}} \boldsymbol{\tau}_{0}\right) \Delta t$. From this, we compute

$$
\mathbf{r}_{\Delta t}^{\prime}=\left(\mathbf{q}_{\nu_{0}}^{\prime}+\mathrm{u}_{2_{0}} \mathbf{q}_{\tau_{0}}\right) \Delta t \boldsymbol{\nu}_{0}+\left(\lambda_{0}+\left(\mathbf{q}_{\tau_{0}}^{\prime}-\mathbf{u}_{2_{0}} \mathbf{q}_{\nu_{0}}\right) \Delta t\right) \boldsymbol{\tau}_{0}
$$

Define for convenience

$$
A=\left(\mathbf{q}_{\nu_{0}}^{\prime}+\mathrm{u}_{2_{0}} \mathbf{q}_{\tau_{0}}\right) \Delta t, \quad B=\left(\lambda_{0}+\left(\mathbf{q}_{\tau_{0}}^{\prime}-\mathrm{u}_{2_{0}} \mathbf{q}_{\nu_{0}}\right) \Delta t\right) .
$$

These quantities are known at every point from the initial data. From [32], we compute

$$
\lambda_{\Delta t}=\left|\mathbf{r}_{\Delta t}^{\prime}\right|=\sqrt{A^{2}+B^{2}},
$$

which gives a rule for updating $\lambda$. Next, from the relation

$$
\boldsymbol{\tau}_{\Delta t}=\mathbf{r}_{\Delta t}^{\prime} / \lambda_{\Delta t}=\frac{A \nu_{0}+B \boldsymbol{\tau}_{0}}{\lambda_{\Delta t}},
$$

we determine that the frames rotate by the angle

$$
\theta=\cos ^{-1}\left(\boldsymbol{\tau}_{\Delta t} \cdot \boldsymbol{\tau}_{0}\right)=\cos ^{-1}\left(B / \lambda_{\Delta t}\right) .
$$

These formulas give exact updates for the frames and stretch $\lambda$, and agree with the continuous case when expanded to first order in $\Delta t$.

Thus far, we have advanced the points, frames, and stretch $\lambda$. The final ingredient needed is a rule to update $\mathbf{u}_{2}$. One option is to recognize that $\mathbf{u}_{2}$ can be obtained from the curvature, which could be computed directly from the updated curve. There are a number of methods to compute the curvature of a discrete curve, see for instance [25]. However, such a method can be computationally expensive and does not utilise the previous data or the mathematical structure inherent in the continuous equations. Instead, the method we propose uses the concept of parallel transport, a familiar concept in differential geometry and used in discrete differential geometry [28]. As stated above, $\mathbf{u}_{2}$ is the rotation of the frames about the binormal along the curve for fixed time. In a discrete setting with frames defined at vertices, $\mathbf{u}_{2}$ is naturally defined on edges as the angle of frame rotation from vertex to vertex divided by the length of the edge. Consider three points at time $\Delta t$, as shown Figure 12. Since the frames at each point are known via the above calculations, the rotation about the binormal from point to point is easily determined. Focusing on point $i$, let $\phi^{-}$denote the angle of rotation from $i-1$ to $i$ and $\phi^{+}$the angle of rotation from $i$ to $i+1$. Then, denoting $\mathbf{u}_{2}^{-}$as the value of $\mathrm{u}_{2}$ on the edge preceeding the point $i$, and $\mathrm{u}_{2}^{+}$for the edge following point $i$, we have

$$
\mathrm{u}_{2}^{-}=\frac{\phi^{-}}{\lambda_{i-1} \Delta \sigma^{-}}, \quad \mathrm{u}_{2}^{+}=\frac{\phi^{+}}{\lambda_{i} \Delta \sigma^{+}},
$$

where $\lambda_{i}$ denotes $\lambda_{\Delta t}\left(\sigma_{i}\right)$, and $\Delta \sigma^{-}, \Delta \sigma^{+}$come from the original discretization of the spatial parameter $\sigma$. Note that $\lambda_{i-1} \Delta \sigma^{-}$is the length of the edge between points $i-1$ and $i$, and similarly for $\lambda_{i} \Delta \sigma^{+}$. To define the value of $\mathrm{u}_{2}$ on the 
vertices where it is needed for the next step, it suffices to take the average, i.e.

$$
\mathrm{u}_{2}\left(\sigma_{i}, \Delta t\right)=\frac{\mathrm{u}_{2}^{-}+\mathrm{u}_{2}^{+}}{2}
$$

an average which is already naturally weighted by the lengths of the edges appearing in the definitions [36].

The formulas [34], [35], and [37] give the rules for updating all local data for given arbitrary velocities.

\section{Appendix: D. Coupling shape evolution to CDRT}

In this appendix we outline the procedure for coupling shape evolution to CDRT in the discrete setting. We take as input an initial curve equipped with frames, stretch $\lambda$, and $\mathrm{u}_{2}$. We also take as known the parameters for coiling $\left(b_{1}\right.$ and $b_{2}$ in Equation [23]), dilation ( $c$ in Equation [25]), rotation ( $b$ in Equation [27]), any global translation $\mathbf{v}_{g}(t)$, and functions for shape evolution $\left(\mathbf{q}_{\nu}(\sigma, t)\right.$ and $\mathbf{q}_{\tau}(\sigma, t)$ from Equation [31]).

Motivated by the notion that shape change can be seen as a secondary layer, the key, computationally, is that we evolve 2 curves: $\mathbf{r}(\sigma, t)$ maps out the "actual" surface, whle $\hat{\mathbf{r}}(\sigma, t)$ stays in the $\mathrm{x}-\mathrm{y}$ plane and only evolves due to shape change, i.e. this curve ignores coiling etc. Along with this, we also must equip each curve with frames, so that along with the frames $(\boldsymbol{\nu}, \boldsymbol{\beta}, \boldsymbol{\tau})$ attached to $\mathbf{r}$, we also have frames $(\hat{\boldsymbol{\nu}}, \hat{\boldsymbol{\beta}}, \hat{\boldsymbol{\tau}})$ attached to $\hat{\mathbf{r}}$. The key observation is that at any time $t$, the curve $\mathbf{r}(\sigma, t)$ will be equivalent to $\hat{\mathbf{r}}(\sigma, t)$ to within translation, rotation, and dilation. Thus, while the frames do not coincide globally, locally they correspond. The idea is illustrated in Figure 13. For each time step, the algorithm works as follows: first, CDRT substeps are completed. For these, only the full curve $\mathbf{r}$ is updated, but to do so, information from $\hat{\mathbf{r}}$ is needed. Recall that coiling is defined by growth in the binormal direction as a linear function along a growth axis. When the shape doesn't change, the velocities and correction terms only need to be determined for the initial step; however once the shape is allowed to evolve the curve $\mathbf{r}$ does not contain the information to define coiling. However, since $\hat{\mathbf{r}}$ remains in the $\mathrm{x}-\mathrm{y}$ plane, the velocity and correction terms are determined in the same way as described in Appendix B. Once the local velocity rules and frame updates are known for $\hat{\mathbf{r}}$, the same rules can be applied for the full curve $\mathbf{r}$. In this way, the value of the local description becomes apparent - there is no need to track global quantities or determine global position, since the growth is fully contained in local variables.

The situation is very similar for dilation and rotation. For dilation, the velocity is in the "radial" direction, meaning locally radial, i.e. the direction outward from the center of the curve. For $\mathbf{r}$, this direction is not trivially determined. It is, however, for $\hat{\mathbf{r}}$. Similarly, for rotation the local circumferential direction is not known on the curve $\mathbf{r}$, but is automatic on $\hat{\mathbf{r}}$. Thus, the local velocity rules are determined via $\hat{\mathbf{r}}$ and the rules described in the Appendix B, and then the same local velocities are applied to the full curve $\mathbf{r}$ while the curve $\hat{\mathbf{r}}$ does not change. Finally, any global translation is trivially accounted for directly on the curve $\mathbf{r}$.

After the CDRT substep is completed, the last substep is to evolve the curves with the shape change functions $\mathbf{q}_{i}(\sigma, t)$. For this substep, both curves and their frames must be updated. First, the curve and frames for $\hat{\mathbf{r}}$ are updated following the approach outlined in the Appendix C. Then, $\mathbf{r}$ and its frames are updated equivalently, with the only difference that a dilation factor must be accounted for in updating $\mathbf{r}$, since aside from dilation, the curves are locally identical. Finally, $\lambda$ and $\mathbf{u}_{2}$ are updated on $\hat{\mathbf{r}}$.

\section{Appendix: E. Mantle stress computation}

In this appendix we compute the stress in the mantle due to adhering to the shell edge. We model the mantle edge as a circular, extensible elastic rod that attaches to the shell edge via a Hookean spring force. Let $r_{s}$ be the radius of the circular shell edge, and let the mantle have radius $a$ in its equilibrium configuration, where $a$ is to be determined. The mantle is assumed to grow according to the function $\gamma(t)$, starting from an initial radius $a_{0}$. Hence, at time $t$ the unstressed radius of the mantle is $\gamma a_{0}$. The axial force $n_{3}{ }^{4}$ in the rod due to compression/stretching depends on the ratio of the actual attached radius $a$ and the unstressed radius $\gamma a_{0}$. Defining

$$
\alpha=\frac{a}{\gamma a_{0}},
$$

the axial force is a function of $\alpha$ that depends on the constitutive properties of the material. We adopt the simplest and most widely used form,

$$
n_{3}=E A(\alpha-1),
$$

where $E$ is the Young's modulus and $A$ is the cross-sectional area, representative of the thickness of the mantle edge. Attachment to the shell edge creates a body force that is a function of the distance between the mantle edge and the shell edge. As this distance is likely to be very small, we use a linear spring force

$$
f=k_{s}\left(a-r_{s}\right),
$$

where $k_{s}$ is a spring stiffness constant, and $f$ points in the radial direction, i.e. the direction normal to the centerline of the rod. Mechanical equilibrium requires that the internal force in the rod balances the attachment body force. In this case, the force balance is

$$
\frac{1}{a} n_{3}+f=0
$$

Inserting $n_{3}$ from [39] into [41] gives a quadratic equation that can be solved for the equilibrium radius. This may be inserted back into [39] to obtain a formula for the stress in the rod due to attachment.

\section{Appendix: F. Simulation parameters}

In this appendix we provide the formulas and growth parameters used to produce the shells simulated in this paper. In terms of CDRT, coiling requires two parameters, $b_{1}$ and $b_{2}$ (see Eq. [23]), dilation and rotation require a single parameter each, $c$ and $b$, as per Eq.'s [25] and [27], respectively, and translation requires a vector $\mathbf{v}_{g}$ (Eq. [30]). Each simulated shell was created with given values for each of these and an aperture shape. The values and shape are provided in Table 1 .

For the simulated shells with ornamentation in Figure 3, a shape changing velocity produced ornamentation. Each spine in Figure 3 a) was produced with a velocity that is spatially Gaussian and sinusoidal in time; that is a spine appearing at time $t_{i}$ with the aperture material point $\sigma_{j}$ was produced with the velocity

$$
\mathbf{v}_{s}=a e^{\left(\left(\sigma-\sigma_{j}\right) / 2 c\right)^{2}} \sin \left(w\left(t-t_{i}\right)\right) \mathbf{s}_{i},
$$

where $\mathbf{s}_{i}$ is the direction of spine growth (given in the plane of the fixed plane curve $\hat{\mathbf{r}})^{5}$. The ornamentation in Figure $3 \mathrm{~b}$ )

\footnotetext{
${ }^{4}$ The notation $n_{3}$ is used to signify the fact that this force acts along the tangent direction of the rod, typically denoted the $\mathbf{d}_{3}$ direction in the theory of elastic rods.

${ }^{5}$ The values of $a, c, w$ varied between different spines.
} 
was produced with a velocity both spatially and temporally sinusoidal,

$$
\mathbf{v}_{s}=(0.2+0.02 \cos (20 \sigma)) \sin \left(16 \pi\left(t-t_{i}\right)\right) \hat{\mathbf{x}} .
$$

For the simulated ammonite in Figure 6, the ribs were produced according to the system [3] with feedback [6], and the stress $n_{3}$ computed according to Appendix E. The parameters used were $a_{0}=1, E A=1, k_{s}=5000, \gamma=1+0.05 t, k_{\phi}=1.2$, $K=40, k_{g}=2$, and $\theta=0$.

For the simulated bivalve in Figure 9, the evolution of the aperture was governed by mechanical energy minimization,

1. H. Moseley. On the geometrical forms of turbinated and discoid shells. Philosophica Transactions of the Royal Society of London, 128:351-370, 1838.

2. M.S. Hutson and X. Ma. Mechanical aspects of developmental biology: perspective on growth and form in the (post)-genomic age. Physical Biology, 5:015001, 2008.

3. G.R. McGhee. Theoretical morphology: the concept and its applications. Columbia Univ $\mathrm{Pr}, 1999$.

4. D.M. Raup. The geometry of coiling in gastropods. Proceedings of the National Academy of Sciences of the United States of America, 47(4):602, 1961.

5. D.M. Raup and A. Michelson. Theoretical morphology of the coiled shell. Science (New York, NY), 147(3663):1294, 1965.

6. E. Savazzi. Biological aspects of theoretical shell morphology. Lethaia, 23(2):195-212, 1990.

7. T. Ubukata. Pattern of growth rate around aperture and shell form in bivalvia: a theoretical morphological study. Paleobiology, 29(4):480, 2003.

8. D.R. Fowler, H. Meinhardt, and P. Prusinkiewicz. Modeling seashells

9. S.H. Rice. The bio-geometry of mollusc shells. Paleobiology, 24(1):133-149, 1998.

10. $\emptyset$. Hammer and $H$. Bucher. Models for the morphogenesis of the molluscan shell. Lethaia, 38(2):111-122, 2005.

11. S. Urdy, N. Goudemand, H. Bucher, and R. Chirat. Allometries and the morphogenesis of the molluscan shell: a quantitative and theoretical model. Journal of Experimenta Zoology Part B: Molecular and Developmental Evolution, 314(4):280-302, 2010.

12. H. Meinhardt and $M$. Klingler. A model for pattern formation on the shells of molluscs. Journal of Theoretical Biology, 126(1):63-89, 1987.

13. A. Boettiger, B. Ermentrout, and G. Oster. The neural origins of shell structure and pattern in aquatic mollusks. Proceedings of the National Academy of Sciences, 106(16):6837, 2009.

14. A. Checa. A model for the morphogenesis of ribs in ammonites inferred from associated microsculptures. Palaeontology, 37(4):863-888, 1994.

15. A.G. Checa and J.S. Crampton. Mechanics of sculpture formation in magadiceramus? rangatira rangatira (inoceramidae, bivalvia) from the upper cretaceous of new zealand. Lethaia, 35(4):279-290, 2002. with the energy the sum of foundation energy and bending energy, Eq.s [7] and [8] respectively. The length excess at each step used was $L-L_{0}=0.25$, other parameters were $E I=0.1, k_{s}=20$.

ACKNOWLEDGMENTS. This publication is based on work supported by Award No. KUK-C1-013-04, made by King Abdullah University of Science and Technology (KAUST) (DEM and AG), and based in part upon work supported by the National Science Foundation under grant DMS-0907773 (AG). AG is a Wolfson/Royal Society Merit Award Holder.

16. $\emptyset$. Hammer. A theory for the formation of commarginal ribs in mollusc shells by regulative oscillation. Journal of Molluscan Studies, 66(3):383, 2000.

17. R. Morita. Finite element analysis of a double membrane tube (dms-tube) and its implication for gastropod shell morphology. Journal of Morphology, 207(1):81-92, 1991.

18. A.G. Checa and A.P. Jimenez-Jimenez. Rib fabrication in ostreoidea and plicatuloidea (bivalvia, pteriomorphia) and its evolutionary significance. Zoomorphology, 122(3):145-159, 2003.

19. D.E. Moulton and A. Goriely. Surface growth kinematics via local curve evolution. 2011.

20. R. Skalak, DA Farrow, and A. Hoger. Kinematics of surface growth. Journal of mathematical biology, 35(8):869-907, 1997.

21. T. Okamoto. Analysis of heteromorph ammonoids by differential geometry. Palaeontology, 31(pt 1):35-52, 1988.

22. S.C. Ackerly. Kinematics of accretionary shell growth, with examples from brachiopods and molluscs. Paleobiology, 15(2):147-164, 1989.

23. E. Savazzi. Geometric and functional constraints on bivalve shell morphology. Lethaia, 20(4):293-306, 1987.

24. J. Tyszka and P. Topa. A new approach to modeling of foraminiferal shells. Paleobiology, 31(3):522, 2005.

25. A.I. Bobenko and Y.B. Suris. Discrete differential geometry: integrable structure Amer Mathematical Society, 2008.

26. P.S. Stevens. Patterns in nature. Little, Brown, 1974.

27. R. Chirat, D.E. Moulton, and A. Goriely. Convergent evolution of spiny mollusc shells points to elastic energy minimum. Preprint.

28. M. Bergou, B. Audoly, E. Vouga, M. Wardetzky, and E. Grinspun. Discrete viscous threads. ACM Transactions on Graphics (TOG), 29(4):1-10, 2010. 
a)
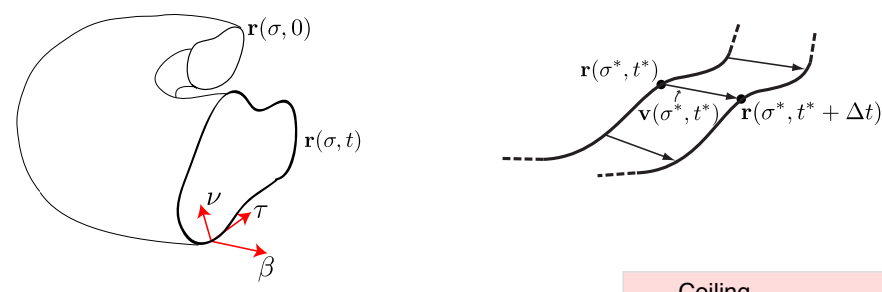

b)
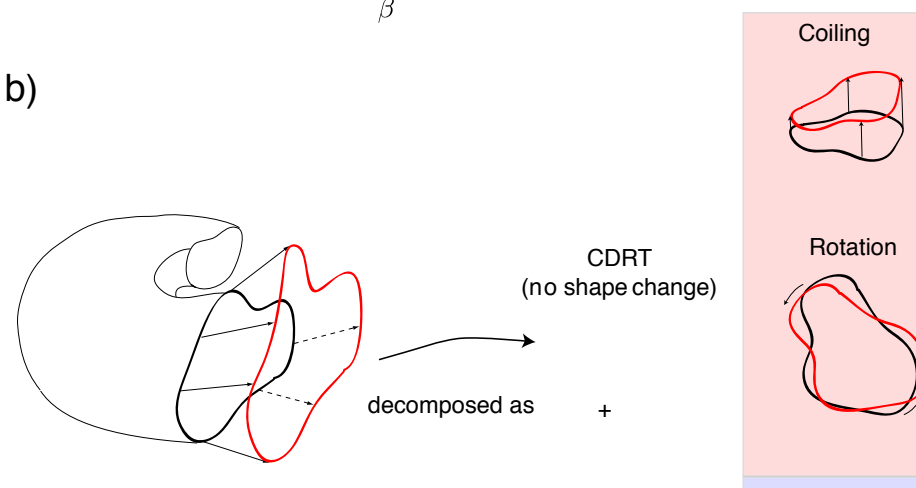

shape change

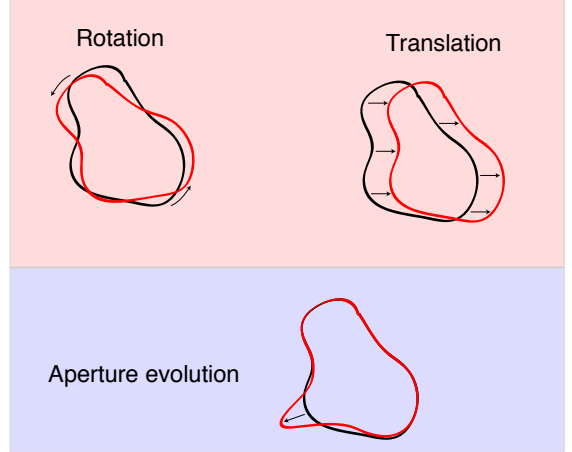

Fig. 1. Setup for modeling shell growth. a) A surface is constructed via the evolution of a generating curve, dictated by a growth velocity field given in terms of a local basis. b) Each growth step is decomposed as a CDRT step, which is described by simple local velocity rules, and an aperture evolution step, which can arbitrarily change the shape of the aperture and is driven by underlying mechanics.

a)

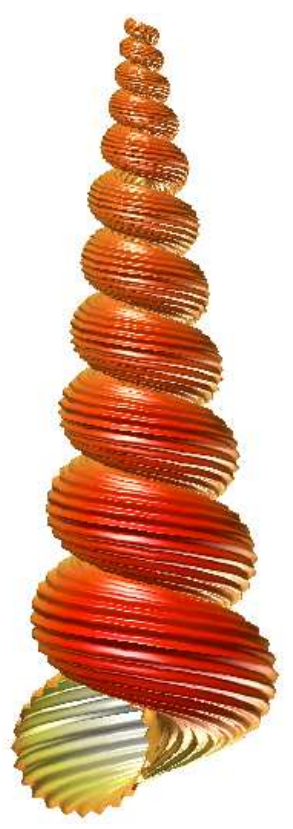

b)

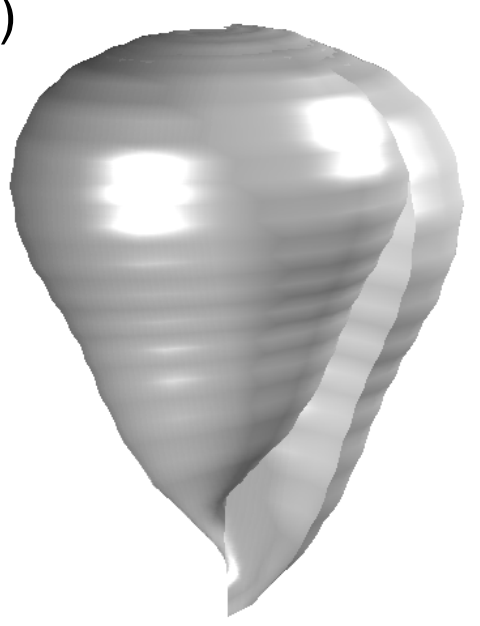

Fig. 2. Simulated seashells with fixed aperture shape constructed through discrete model. Parameter values are given in Appendix F. 
a)
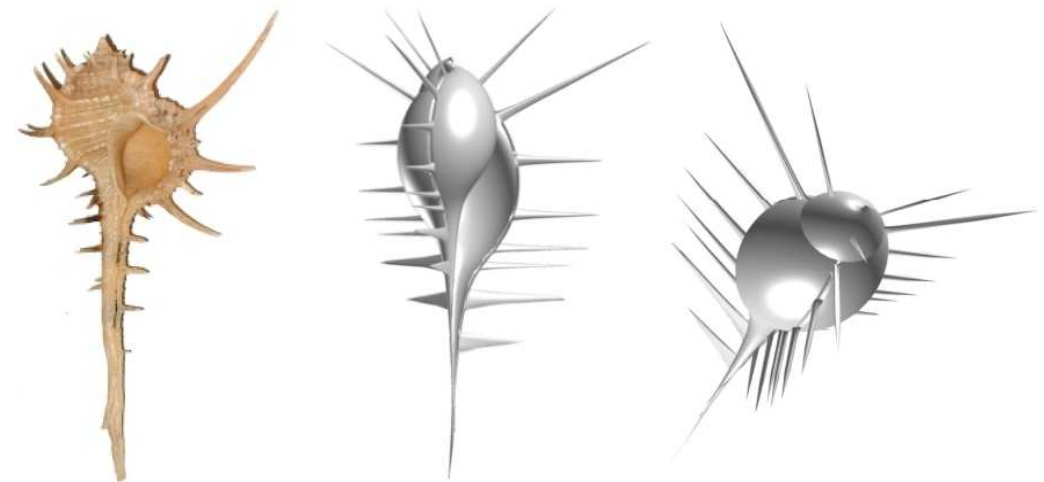

Murex tenuirostrum
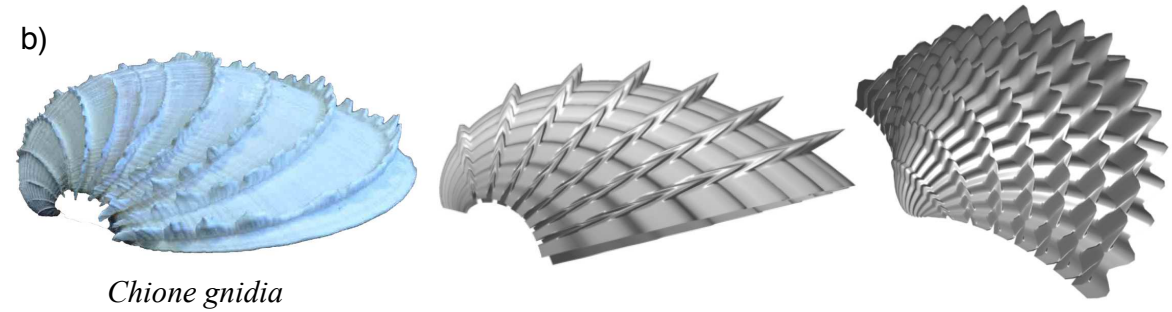

Chione gnidia

Fig. 3. Samples of shells simulated with a shape change function to produce ornamentation layered on top of fixed coiling, dilation, rotation, and translation velocities. Parameter values and shape change functions are given in Appendix F.
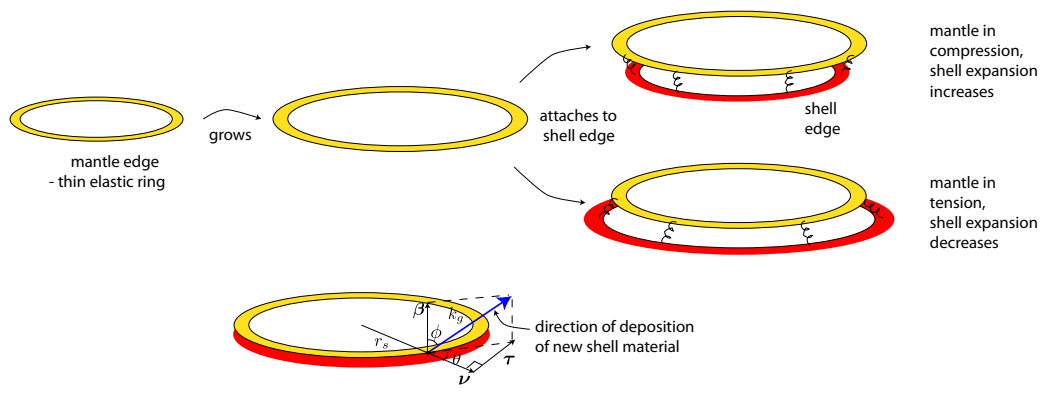

Fig. 4. Setup for the mantle growth model to produce commarginal ribs. 
a)

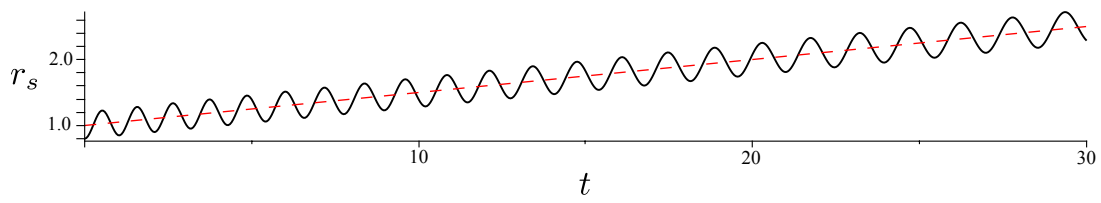

b)

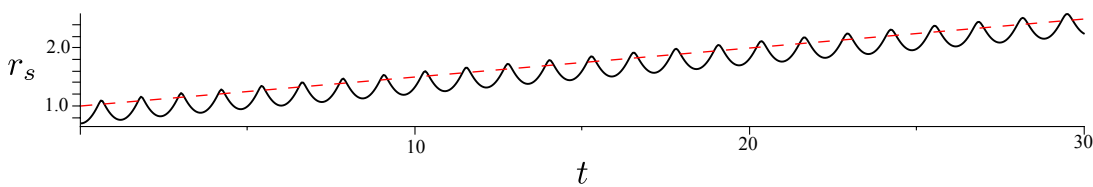

c)

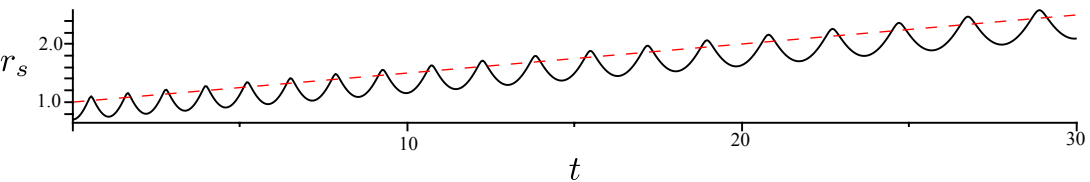

Fig. 5. Solutions to the system [3]. Shell edge radius plotted against time for a) linear feedback model, Equation [4] with $k_{\phi}=15$, b) nonlinear feedback, Equation [5] with $k_{\phi}=1.2, K=40$, and c) decreasing nonlinear feedback, Eq. [6] with $k_{\phi}=1.2, K=40$. The dashed red line is the unstressed radius of the growing mantle. Other parameters are $r_{s}(0)=0.8$ in a), $r_{s}(0)=0.7$ in b) and c), and in all cases $\phi(0)=0, \gamma=1+0.05 t, E A=1, k_{s}=5000$, and $k_{g}=2$.
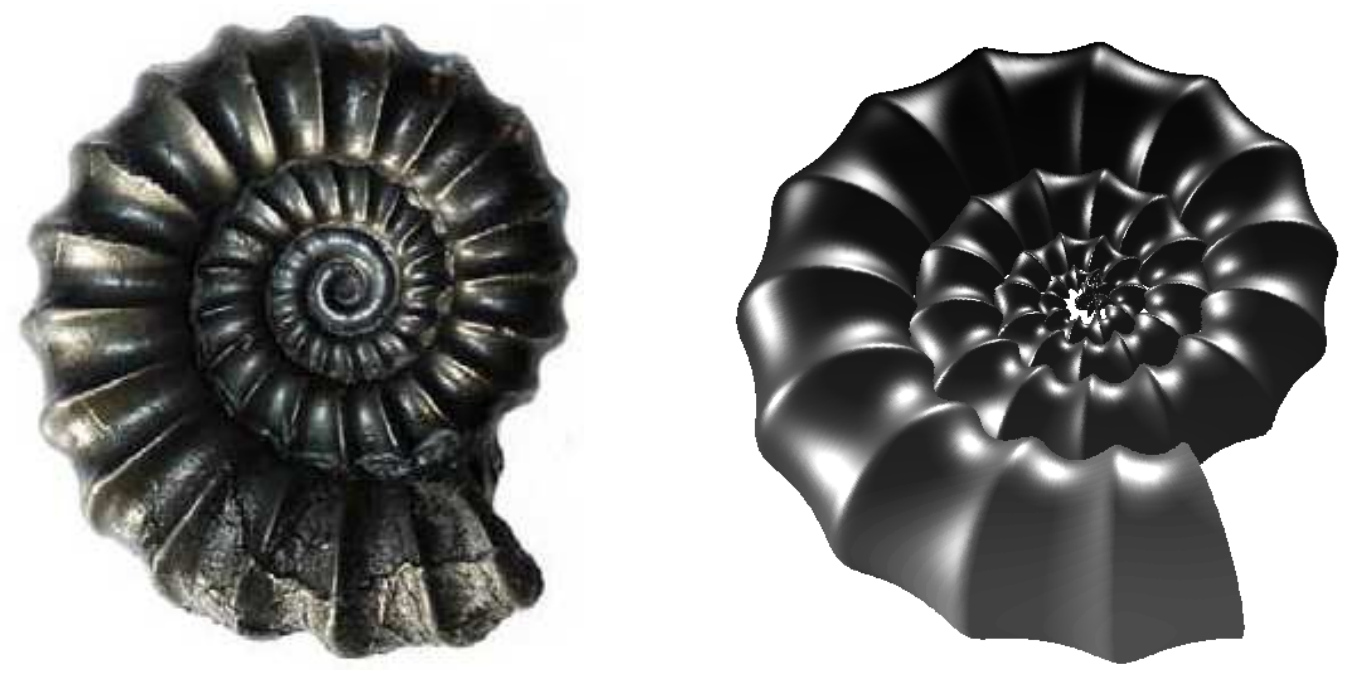

Fig. 6. Simulation of an ammonite. Stress in the mantle governs the dilation of the shell edge, and is combined with a fixed rule for coiling. Parameter values are given in Appendix F. 


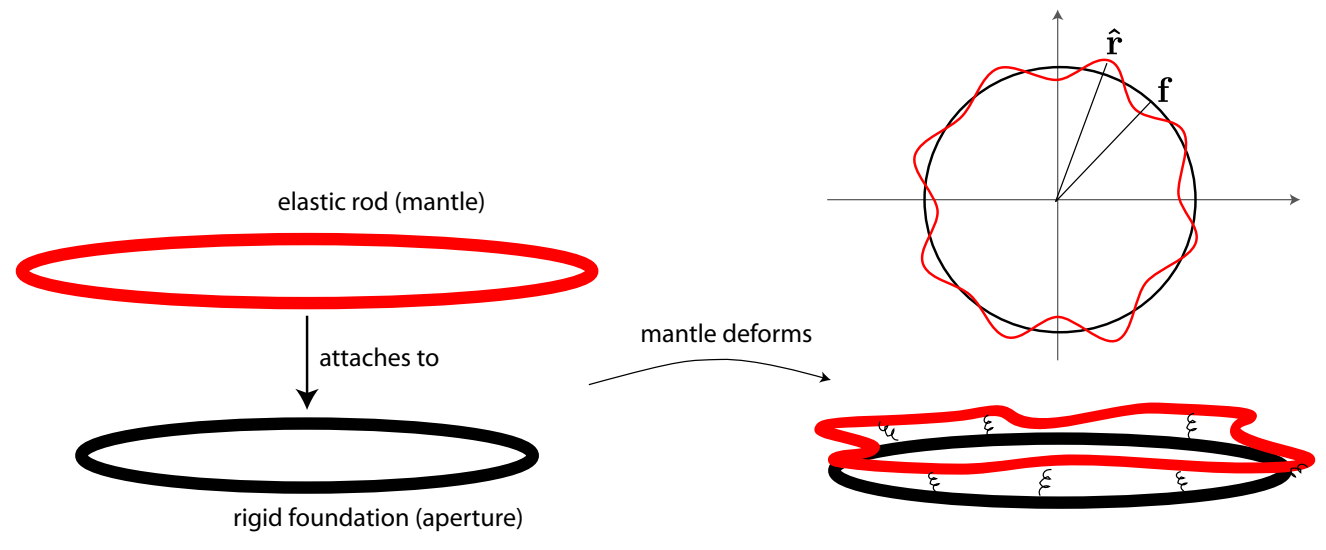

Fig. 7. Setup for mantle buckling model. The shape of the deformed mantle, described by the curve $\hat{\mathbf{r}}$, is found as the minimizer of the sum of bending energy and spring energy due to attachment to the foundation, given by the curve $\mathbf{f}$.

I.a

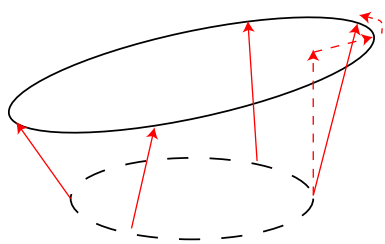

\section{II.a}

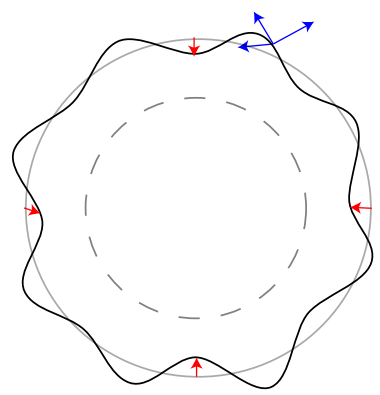

I.b

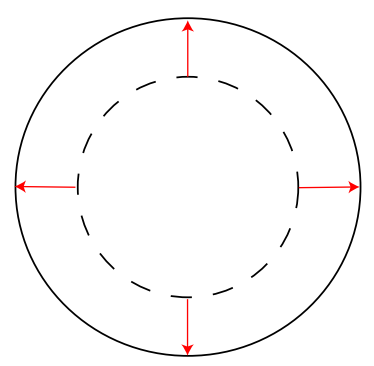

II.b

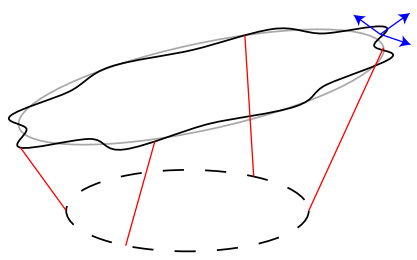

Fig. 8. The computational steps to simulate mantle buckling with shell evolution. Each growth step consists of two substeps. In the first substep, CDRT is applied to the full curve (la) while only dilation is applied to the fixed plane curve (Ib). In the second substep, the shape of the fixed plane curve is updated (Ila) by computing the mechanical deformation of the mantle, after which the full curve is accordingly updated using the local frame correspondence. 

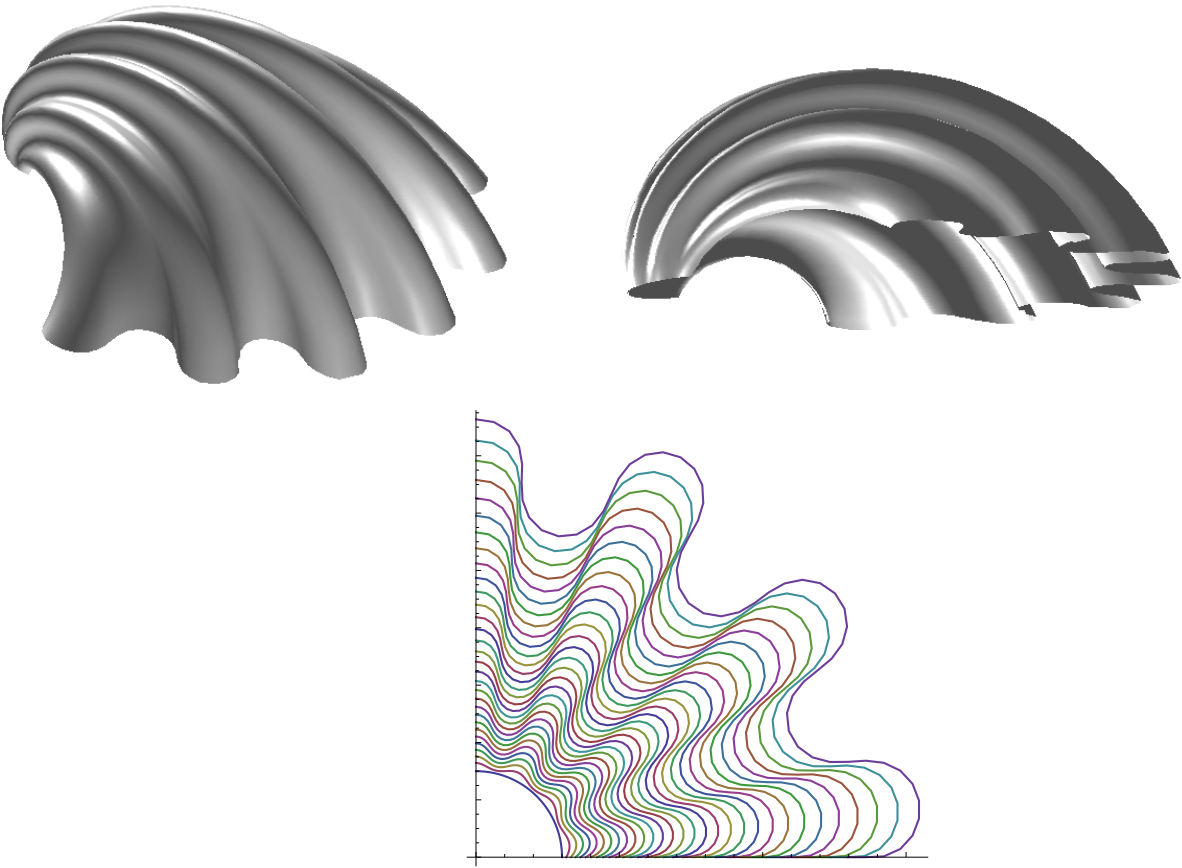

Fig. 9. Simulation of the growth of a bivalve shell. Mechanical deformation of the mantle dictates the evolution of the aperture shape, and is combined with parameters for coiling, dilation, and translation. Parameter values are given in Appendix F.

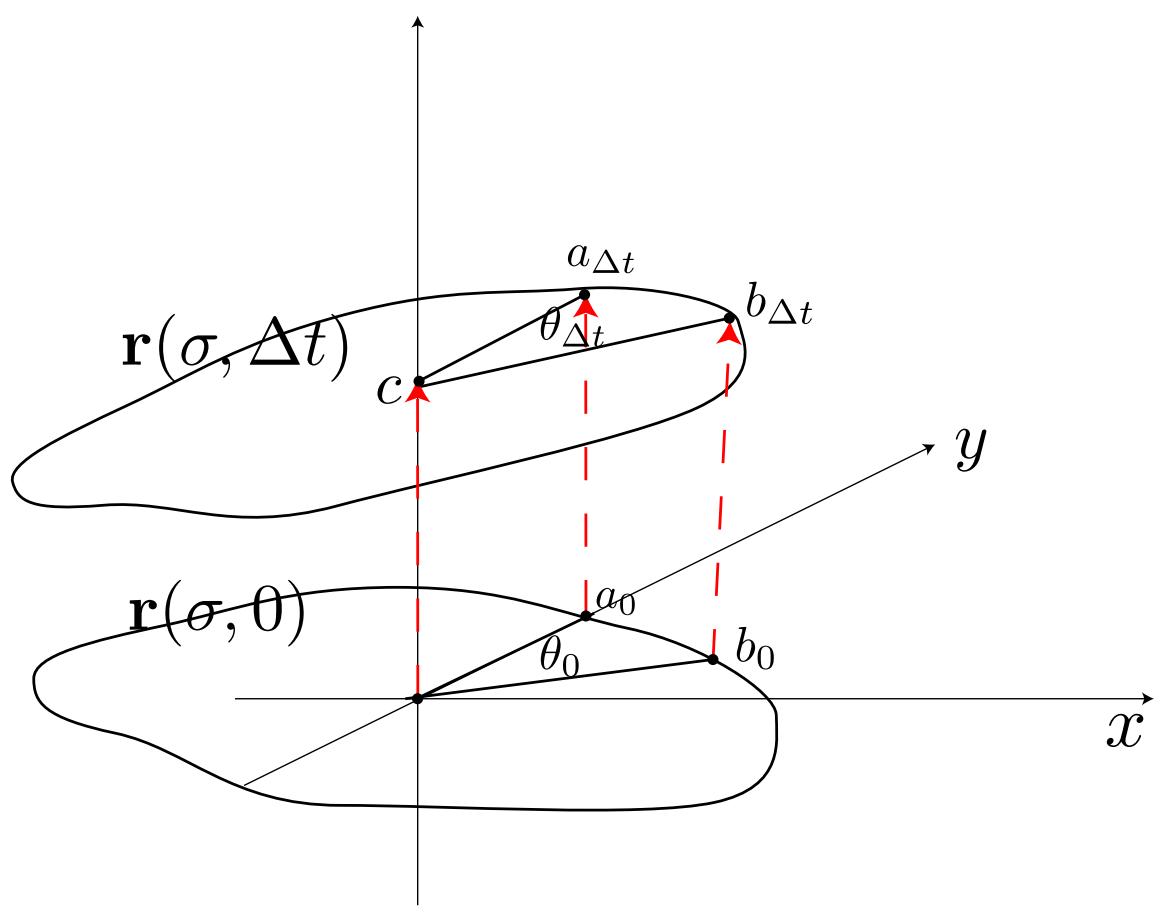

Fig. 10. Discrete coiling schematic. The point $a_{0}$ and the origin move only in the binormal direction, and are used to determine the correction velocities for all other points. 


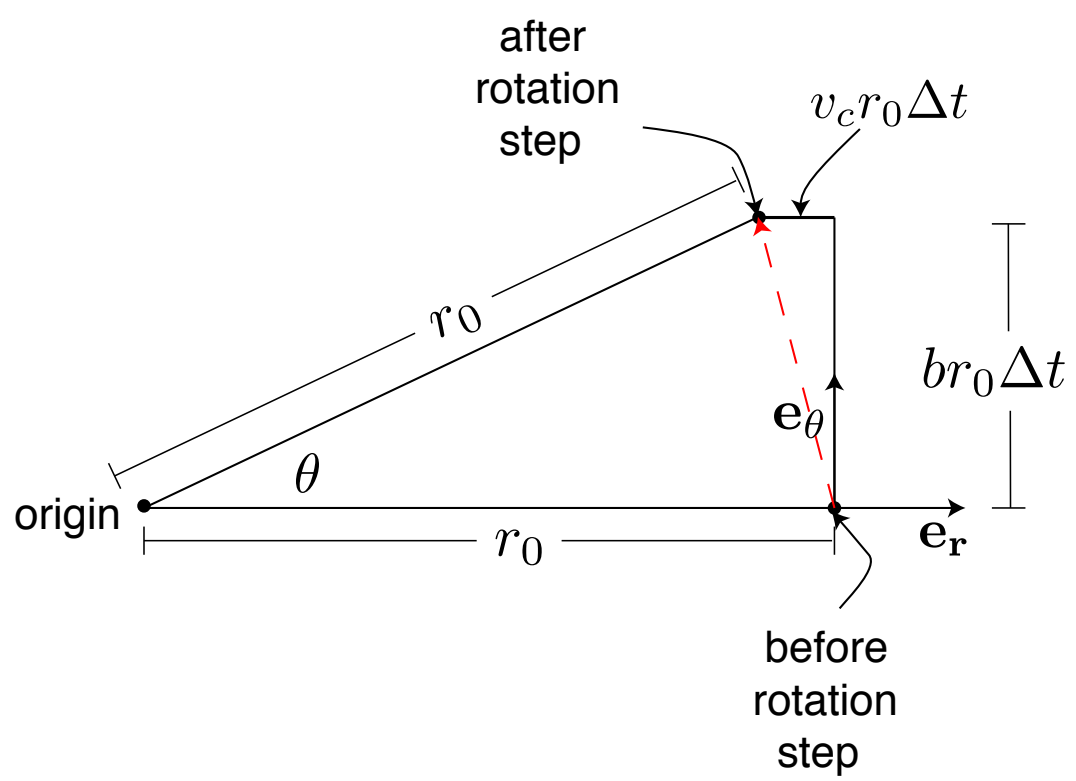

Fig. 11. Discrete rotation. A correction velocity of magnitude $v_{c} r_{0} \Delta t$ must be added in the radial direction.

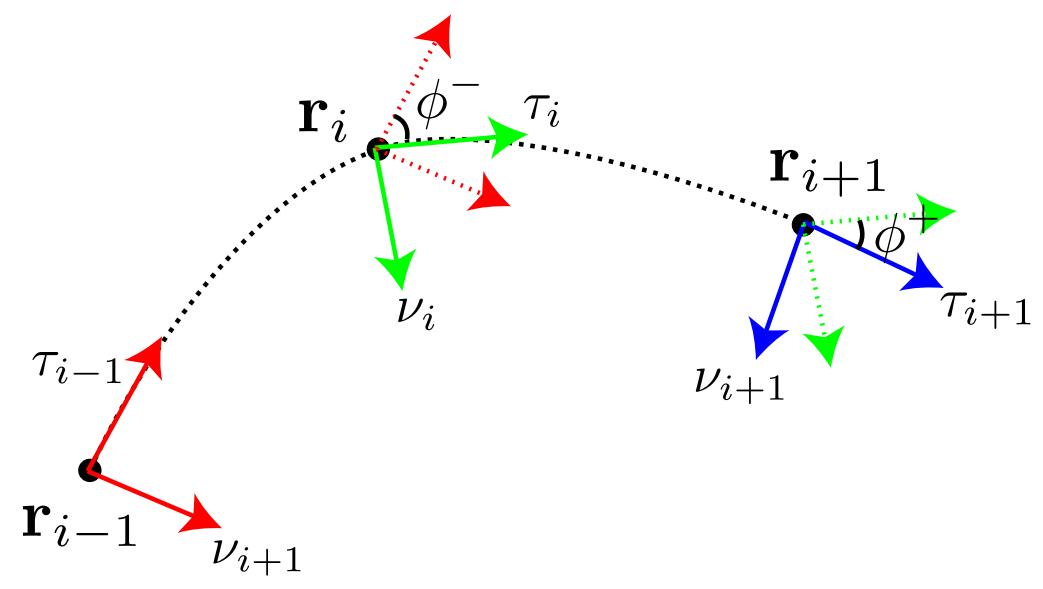

Fig. 12. Schematic for computation of $\mathbf{u}_{2}$ in discrete curve evolution. Curvature, and hence $\mathrm{u}_{2}$ are determined by the rotation of frames from vertex to vertex and the length of edges. 

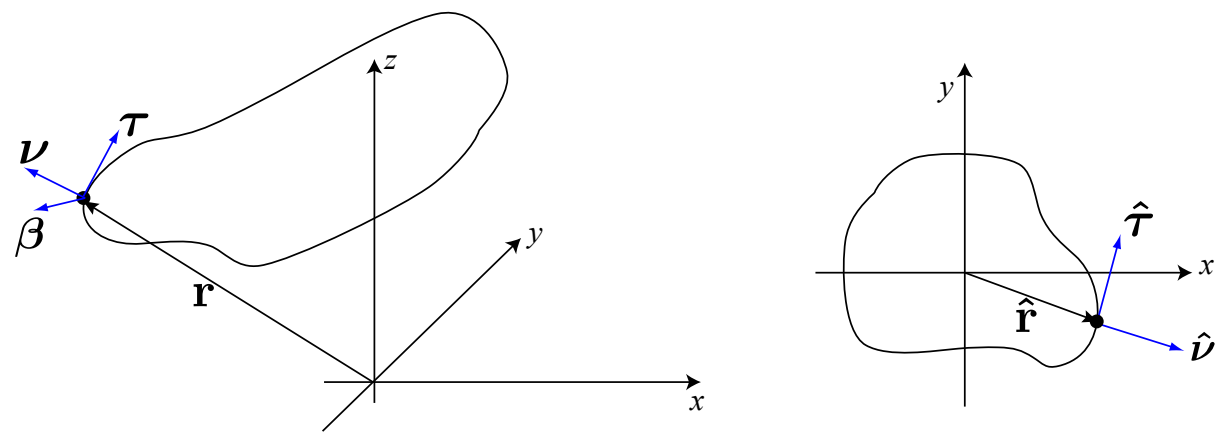

Fig. 13. To couple shape change with CDRT, 2 versions of the curve are evolved: the full version $\mathbf{r}$ (left) and a reference curve $\hat{\mathbf{r}}$ that remains in the $x-y$ plane and only tracks shape change.

Table 1. CDRT parameter values

\begin{tabular}{|c|c|c|c|c|c|}
\hline Figure & Shape & $\begin{array}{c}\text { Coiling } \\
b_{1}, b_{2} \\
\end{array}$ & $\begin{array}{c}\text { Dilation } \\
c \\
\end{array}$ & $\begin{array}{c}\text { Rotation } \\
b \\
\end{array}$ & $\begin{array}{c}\text { Translation } \\
\mathbf{v}_{g} \\
\end{array}$ \\
\hline $2 a$ & & 1,10 & 0.25 & 0 & {$[0,1 / \pi, 0]$} \\
\hline $2 b$ & & $0.1,8$ & 0.3 & 0 & {$[0,-0.6,0]$} \\
\hline $3 a$ & & $0.1,-1.5$ & 0.1 & 0 & {$[0,-0.1,0]$} \\
\hline $3 b$ & & $0.1,5+0.5 t$ & 5 & 0 & {$[0,0,0]$} \\
\hline 6 & & $0.75,0.351$ & NA & 0 & {$[0,0,0]$} \\
\hline 9 & & $0.25,0.116$ & 0.05 & 0 & {$[0,-0.15,0]$} \\
\hline
\end{tabular}





\section{RECENT REPORTS}

51/11 Wound healing angiogenesis: the clinical implications of a simple mathematical model

$\mathrm{Du}$

Gunzburger

Lehoucq

Zhou

März

Moulton

Goriely

Hennessey

Münch

Luo

Majumdar

Erban

56/11 Real-Time Fluid Effects on Surfaces using the Closest Point Method

$57 / 11$ Isolating intrinsic noise sources in a stochastic genetic switch

58/11 Riemann-Cartan Geometry of Nonlinear Dislocation Mechanics

59/11 Helices through 3 or 4 points?

60/11 Bayesian data assimilation in shape registration

61/11 Asymptotic solution of a model for bilayer organic diodes and solar cells

62/11 Neural field model of binocular rivalry waves

63/11 Front propagation in stochastic neural fields

64/11 Stability estimates for a twisted rod under terminal loads: a threedimensional study

65/11 Adaptive Finite Element Method Assisted by Stochastic Simulation of Chemical Systems
Auer

Macdonald

Treib

Schneider

Westermann

Newby

Yavari

Goriely

Goriely

Neukirch

Hausrath

Cotter

Cotter

Vialard

Richardson

Please

Kirkpatrick

Bressloff

Webber

Bressloff

Webber

Majumdar

Prior

Goriely

Cotter

Vejchodsky

Erban

Prior

Berger 
$67 / 11$ Tear film thickness variations and the role of the tear meniscus

Please

Fulford

Fulford

Collins

68/11 Comment on "Frequency-dependent dispersion in porous media"

Davit

Quintard

69/11 Molecular Tilt on Monolayer-Protected Nanoparticles

Giomi

Bowick

Ma

Majumdar

70/11 The Capillary Interaction Between Two Vertical Cylinders

Cooray

Cicuta

Vella

71/11 Nonuniqueness in a minimal model for cell motility

Gallimore

Whiteley

Waters

King

Oliver

72/11 Symmetry of uniaxial global Landau-de Gennes minimizers in the

Henao theory of nematic liquid crystals

Majumdar

73/11 Filling of a Poisson trap by a population of random intermittent

Bressloff searchers

Newby

Copies of these, and any other OCCAM reports can be obtained from:

Oxford Centre for Collaborative Applied Mathematics Mathematical Institute

24 - 29 St Giles'

Oxford

OX1 3LB

England

www.maths.ox.ac.uk/occam 